\title{
Outcome after Emergency Surgery for Acute Perforated Diverticulitis in 200 Cases
}

\author{
Jefrey Vermeulen ${ }^{\mathrm{a}} \quad$ George P. Akkersdijk $^{\mathrm{b}}$ Martijn P. Gosselink ${ }^{\mathrm{a}}$ \\ Wim C.J. Hop ${ }^{c}$ Guido H. Mannaerts ${ }^{d}$ Erwin van der Harst ${ }^{a}$ \\ Peter-Paul L.O. Coene ${ }^{a}$ Wibo F. Weidema $^{b}$ Johan F. Lange ${ }^{e}$ \\ ${ }^{a}$ Department of Surgery, MCRZ St. Clara Hospital and Zuider Hospital, Rotterdam, ${ }^{\mathrm{b}}$ Department of Surgery, \\ Ikazia Hospital, Rotterdam, 'Department of Epidemiology and Biostatistics, EMC Rotterdam, Rotterdam, \\ ${ }^{\mathrm{d} D e p a r t m e n t}$ of Surgery, Sint Franciscus Hospital, Rotterdam, and e Department of Surgery, EMC Rotterdam, \\ Rotterdam, The Netherlands
}

\section{Key Words \\ Perforated diverticulitis - Hartmann's procedure • Primary anastomosis • Mortality • Reintervention • Surgeon's experience}

\begin{abstract}
Background: Mortality and morbidity rates of acute perforated diverticulitis remain high. The ideal treatment is still controversial. The object of this study was to compare patients with perforated diverticulitis treated either by resection with primary anastomosis (PA) or Hartmann's procedure (HP). Methods: A multicenter study was carried out on 200 consecutive patients with acute perforated diverticulitis who were presented in the surgical units of four affiliated teaching hospitals in Rotterdam, The Netherlands, between 1995 and 2005. Mortality and morbidity were compared in relation to type of surgery, ASA classification, age, gender, Mannheim Peritonitis Index (MPI), Hinchey score, surgeon's experience, and the time of operation. Results: There was a tendency for more severely affected patients (Hinchey, MPI, ASA and age) to undergo HP. Multivariate logistic regression analysis showed no significant difference in mortality between HP and PA. After HP, more patients needed one or
\end{abstract}

\section{KARGER}

Fax +41613061234

E-Mail karger@karger.ch

www.karger.com (c) 2007 S. Karger AG, Basel

$0253-4886 / 07 / 0245-0361 \$ 23.50 / 0$

Accessible online at:

www.karger.com/dsu more reinterventions to treat postoperative complications compared to PA. Besides, HP resulted in a longer total hospital and intensive care unit stay. Specialist colorectal surgeons performed significantly more frequently a PA instead of a HP and had fewer postoperative complications than general surgeons. The time of operation did not influence the choice of surgical procedure. Conclusion: Selected patients with perforated diverticulitis can be managed well by PA, as it does not seem to be inferior to HP in terms of severe postoperative complications that need surgical or radiological reintervention and mortality. This decision should be made while taking into account the patient's concomitant diseases, response on preoperative resuscitation and the availability of a surgeon experienced in colorectal surgery.

Copyright $\odot 2007$ S. Karger AG, Basel

\section{Introduction}

Diverticular disease has emerged as a common problem in Western countries over the course of the 20th century. Up to two-thirds of individuals are affected with diverticulosis by the age of 70 [1] and admission rates for diverticular disease are still increasing in the aged popu- 
lation [2]. Nearly a quarter of the patients require an emergency operation because of perforation, peritonitis, or systematic complications [3].

Regardless of selected strategy, emergency operations for diverticular disease are associated with substantial morbidity and mortality. Therefore the optimal treatment for complicated diverticulitis is still a matter of debate [4]. The current controversy is whether resection with primary anastomosis (PA) is safe or if a Hartmann's procedure (HP) still remains standard practice in case of generalized peritonitis complicating diverticulitis. For many surgeons, HP still remains the favored option in these patients [5]. Improvements in surgical techniques, radiological intervention techniques, anesthesia, advances in intensive care medicine and progress in the management of peritoneal sepsis have led to an increasing interest in PA with or without diverting stoma or colonic lavage [6-8].

The object of this study was to compare the mortality rates, the incidence of re-operations or additional interventions and length of hospital stay in patients with perforated diverticulitis treated by HP and PA in relation to the patient's characteristics, severity of the disease, surgeon's experience, and the time of operation.

\section{Patients and Methods}

All consecutive patients who underwent HP or PA between 1995 and 2005 for acute perforated sigmoid diverticulitis in the surgical units of four affiliated teaching hospitals in Rotterdam, The Netherlands (St. Clara Hospital, Zuider Hospital, Sint Franciscus Hospital and Ikazia Hospital) were included in this study. The indications for surgery were clinical signs of diffuse peritonitis or presence of septic status with acute abdominal pain, free gas on plain abdominal radiography or specific findings at ultrasonography or computerized tomography. All but 5 patients were operated within $24 \mathrm{~h}$ and received pre- and postoperative broadspectrum intravenous antibiotics. In none of the patients was a preoperative bowel preparation used.

139 patients underwent $\mathrm{HP}$ and 61 patients underwent $\mathrm{PA}$. Colon resections for perforated diverticulitis in the PA group were sigmoid resection (51), left hemicolectomy (8) and anterior resection (2). In the PA group, 16 patients $(26 \%)$ received a diverting ileostomy. The decision to perform one or the other procedure was left to the discretion of the surgeon on call. The operation was performed 84 times by a colorectal specialist surgeon and 116 times by a general surgeon.

In this study, all additional radiological interventions or reoperations after primary emergency surgery were mentioned as reinterventions. Reinterventions were defined as radiological-assisted percutaneous drainage of abdominal or pelvic abcess, open abdominal wound management or re-operations because of ongoing sepsis, abdominal abcess, evisceration, anastomotic leakage or stoma-related complications.
A prospective computerized morbidity and mortality registration was carried out for all patients admitted to and operated in the surgical departments of all four hospitals. Patients who underwent emergency surgery for acute perforated diverticulitis could therefore be identified. Severity of disease was stratified with the Mannheim Peritonitis Index (MPI) [9] and Hinchey score [10]. The MPI was retrospectively calculated from the medical reports of all patients, whereas the Hinchey score was distracted from the surgical reports. Patients with generalized peritonitis underwent a HP 95 times (Hinchey III, $\mathrm{n}=62$; Hinchey IV, $\mathrm{n}=33$ ), whereas only 26 patients underwent PA (Hinchey III, $\mathrm{n}=$ 21; Hinchey IV, $n=5$ ). Age, gender, American Society of Anesthesiologist (ASA) classification, severity of disease, postoperative mortality (30 days), number of reinterventions, surgeon's experience in colorectal surgery, time of operation, hospital stay and stay on the intensive care unit in the two groups were compared.

\section{Statistics}

Data are represented as mean \pm SD or median \pm SD unless indicated otherwise. Comparisons between the two groups were made with Mann-Whitney tests for quantitative variables or graded outcomes and the Fisher's exact test for categorical data. A multivariate logistic regression analysis of the significant clinical variables in univariate analysis was done using logistic regression models with mortality or required reintervention as the outcome. Differences were considered significant at a two-tailed $\mathrm{p}$ value of $<0.05$.

\section{Results}

A total of 200 patients underwent an emergency operation between January 1995 and January 2005. During this period the percentages of $\mathrm{HP}$ and PA, which were performed each year, did not change with time $(\mathrm{p}=0.82)$. Patients' characteristics are shown in table 1 . No differences in surgical procedure or baseline patient characteristics were noted between the series from the different hospitals. Patients who underwent HP were significantly older and showed higher Hinchey scores, higher ASA classifications and MPI. Specialist colorectal surgeons performed significantly more frequently a PA instead of HP. There was no relation found between time of operation and the type of surgical procedure $(\mathrm{p}=0.66) .16 \mathrm{PA}$ patients $(26 \%)$ received a temporary ileostomy. No differences were found between PA patients with or without a temporary ileostomy.

\section{Mortality}

Total mortality was $27 \%$ after emergency surgery for perforated surgery. 47 patients died after HP compared to 7 PA patients ( 34 vs. $11 \%$, respectively; $\mathrm{p}<0.01$ ). Mortality seemed related to type of surgery, age, ASA classification, Hinchey score, MPI and surgeon's experience (table 2). 
Table 1. Patients' characteristics

\begin{tabular}{|c|c|c|c|}
\hline & $\begin{array}{l}\text { Hartmann's } \\
\text { procedure }\end{array}$ & $\begin{array}{l}\text { Primary } \\
\text { anastomosis }\end{array}$ & $\mathrm{p}$ \\
\hline Patients & $139(70)$ & $61(30)$ & \\
\hline Age $^{1}$, years & $69 \pm 13$ & $62 \pm 15$ & $<0.01$ \\
\hline Sex & & & $0.54 \mathrm{NS}$ \\
\hline Male (94) & $64(46)$ & $25(41)$ & \\
\hline Female (113) & $75(54)$ & $36(59)$ & \\
\hline Hinchey score ${ }^{1}$ & & & 0.01 \\
\hline I (36) & $26(19)$ & $9(15)$ & \\
\hline II (44) & $18(13)$ & $26(43)$ & \\
\hline III (88) & $62(45)$ & $21(34)$ & \\
\hline IV (39) & $33(24)$ & $5(8)$ & \\
\hline ASA classification ${ }^{1}$ & & & $<0.01$ \\
\hline I (43) & $25(18)$ & $17(28)$ & \\
\hline II (53) & $31(22)$ & $19(31)$ & \\
\hline III (66) & $46(33)$ & $18(30)$ & \\
\hline IV (45) & 37 (27) & $7(11)$ & \\
\hline $\mathrm{MPI}^{2}$ (points) & $21 \pm 8.0$ & $17 \pm 6.0$ & $<0.01$ \\
\hline Time of operation & & & $0.66 \mathrm{NS}$ \\
\hline During office hours (134) & $89(64)$ & $38(62)$ & \\
\hline Outside office hours (73) & $50(36)$ & $23(38)$ & \\
\hline Surgeon's experience & & & 0.01 \\
\hline General surgeon (116) & $89(64)$ & $27(45)$ & \\
\hline Colorectal surgeon (84) & $50(36)$ & $34(55)$ & \\
\hline
\end{tabular}

Values in parentheses are percentages. NS = Not significant.

${ }^{1}$ Mean age, Hinchey score, American Society of Anesthesiologist (ASA) classification $\pm \mathrm{SD}$.

${ }^{2}$ Median Mannheim Peritonitis Index \pm SD.

Because of selection bias, patients who had undergone HP were significantly older, had more comorbidities, had a more severe disease and were more frequently operated by specialist colorectal surgeons, a multivariate logistic regression analysis was performed to compare both groups. After adjustment for these risk factors, mortalities in both groups did not differ significantly (OR 2.1; 95\% CI 0.8-4.8; $\mathrm{p}=0.15$ ). Age, MPI and ASA classification were still significantly related to a higher mortality rate $(\mathrm{p}<0.01, \mathrm{p}<0.01$ and $\mathrm{p}<0.01$ respectively) as shown in table 3.

The performance of a diverting stoma in the PA group did not show a significant advantage over PA without diverting stoma (mortality of 6 and 13\%, respectively; $\mathrm{p}=$ $0.66)$ after univariate analysis. These groups were too small ( $\mathrm{n}=16$ and 45 , respectively) for multivariate logistic regression analysis.

During the study, period postoperative mortality rates remained constant $(p=0.18)$. There were no significant differences in mortality between the four centers $(\mathrm{p}=$ $0.49)$.

Emergency Surgery for Acute Perforated Diverticulitis
Table 2. Univariate analysis for relation between the listed variables and postoperative death (within 30 days) and the need for reinterventions

\begin{tabular}{lcl}
\hline & $\begin{array}{l}\text { Mortality } \\
\mathrm{p}\end{array}$ & $\begin{array}{l}\text { Reinterventions } \\
\mathrm{p}\end{array}$ \\
\hline Surgical procedure & $<0.01$ & $<0.01$ \\
Age & $<0.01$ & $0.394 \mathrm{NS}$ \\
Sex & $1 \mathrm{NS}$ & $1 \mathrm{NS}$ \\
ASA classification & $<0.01$ & $<0.01$ \\
Hinchey score & 0.012 & $<0.01$ \\
Mannheim Peritonitis Index & $<0.01$ & $<0.01$ \\
Time of operation & $0.370 \mathrm{NS}$ & $0.220 \mathrm{NS}$ \\
Surgeon's experience & 0.046 & $<0.01$ \\
\hline
\end{tabular}

NS = Not significant.

\section{Reinterventions}

To treat their (severe) postoperative complications or sepsis, 54 patients (27\%) needed one or more re-operations or additional (radiological) interventions (HP, $\mathrm{n}=$

Dig Surg 2007;24:361-366 
Table 3. Multivariate logistic regression analysis for postoperative death within 30 days

\begin{tabular}{lllr}
\hline & \multicolumn{3}{c}{ Postoperative mortality } \\
\cline { 2 - 4 } & OR & $95 \%$ CI & \multicolumn{1}{c}{$\mathrm{p}$} \\
\hline HP vs. PA & 2.1 & $0.8-4.8$ & 0.15 \\
Age & 1.2 & $1.1-1.3$ & $<0.01$ \\
ASA (vs. ASA I) & & & $<0.01$ \\
$\quad$ II & 2.2 & $0.6-9.3$ & 0.29 \\
$\quad$ III & 5.8 & $4.7-22.7$ & $<0.01$ \\
IV & 9.8 & $2.5-39.5$ & $<0.01$ \\
Hinchey (vs. Hinchey I) & & & 0.06 \\
$\quad$ II & 1.5 & $0.5-5.7$ & 0.52 \\
$\quad$ III & 1.2 & $0.4-3.7$ & 0.78 \\
IV & 3.9 & $1.0-13.8$ & 0.03 \\
MPI & 1.4 & $1.2-1.5$ & $<0.01$ \\
Surgeon's experience & 1.3 & $0.7-2.7$ & 0.45 \\
\hline
\end{tabular}

$\mathrm{OR}=$ Odds ratio $\mathrm{HP}=$ Hartmann's procedure $\mathrm{PA}=$ resection with primary anastomosis; ASA = American Society of Anesthesiologist classification; MPI = Mannheim Peritonitis Index.

$46 ; 33 \%, \mathrm{PA}, \mathrm{n}=8 ; 13 \%, \mathrm{p}<0.01)$. Table 2 shows whether or not the need for radiological or surgical reinterventions was related to the listed factors.

Multivariate analysis, adjusting for Hinchey score, ASA, age, MPI and surgeon's experience, showed that HP necessitated significantly more frequent reinterventions compared to PA (OR 2.4; 95\% CI 1.2-5.7; p < 0.05). Higher ASA classification, Hinchey score, MPI and surgeon's experience were also significantly related with need for reinterventions after primary surgery (table 4 ). When comparing the need for reinterventions in high-risk patients (older age, generalized peritonitis, more comorbidities and higher MPI) between HP and PA groups, no significant differences could be found, as shown in table 5 .

Within the PA group, univariate analysis showed no significant differences found in the number of complications that needed reintervention whether or not a diverting ileostomy was performed ( $19 \mathrm{vs.} 11 \%$ respectively; $\mathrm{p}=$ 0.42 ). Three patients (5\%) developed an anastomotic leakage after PA; 1 of them had received a diverting ileostomy during primary surgery and anastomotic leakage could be treated conservatively. The other patients needed reoperation because of their anastomotic leakage. There were no significant differences in number of reinterventions between the four centers $(\mathrm{p}=0.77)$.
Table 4. Multivariate logistic regression analysis for the need of re-operation or other additional interventions after surgery

\begin{tabular}{llcr}
\hline & \multicolumn{3}{c}{ Postoperative need for reinterventions } \\
\cline { 2 - 4 } & OR & $95 \%$ CI & \multicolumn{1}{c}{$\mathrm{p}$} \\
\hline HP vs. PA & 2.4 & $1.2-5.7$ & 0.05 \\
Age & 1.0 & $0.96-1.01$ & 0.24 \\
ASA (vs. ASA I) & & & $<0.01$ \\
$\quad$ II & 1.2 & $0.4-3.7$ & 0.81 \\
III & 6.4 & $2.2-18.3$ & $<0.01$ \\
IV & 2.6 & $0.8-8.1$ & 0.10 \\
Hinchey (vs. Hinchey I) & & & 0.02 \\
$\quad$ II & 1.0 & $0.3-3.3$ & 0.98 \\
$\quad$ III & 1.0 & $0.3-2.9$ & 0.98 \\
IV & 3.9 & $1.3-12.7$ & 0.02 \\
MPI & 1.1 & $1.0-1.2$ & $<0.01$ \\
Surgeon's experience & 4.9 & $2.5-9.7$ & 0.04 \\
\hline
\end{tabular}

$\mathrm{OR}=$ Odds ratio; $\mathrm{HP}=$ Hartmann's procedure; $\mathrm{PA}=$ resection with primary anastomosis; ASA = American Society of Anesthesiologist classification; MPI = Mannheim Peritonitis Index.

Table 5. The need for reinterventions in high-risk patients

\begin{tabular}{llll}
\hline Risk factor & $\begin{array}{l}\text { Hartmann's } \\
\text { procedure } \\
\mathrm{n}=48\end{array}$ & $\begin{array}{l}\text { Primary } \\
\text { anastomosis } \\
\mathrm{n}=8\end{array}$ & $\mathrm{p}$ \\
\hline Age $\geq 70$ years & $24(50)$ & $4(50)$ & 1.0 \\
Hinchey score III and IV & $35(73)$ & $4(50)$ & 0.23 \\
ASA $\geq$ III & $34(71)$ & $6(75)$ & 1.0 \\
MPI $\geq 26$ & $25(52)$ & $2(25)$ & 0.25 \\
Two or more risk factors & $37(77)$ & $5(63)$ & 0.40 \\
Three or more risk factors & $24(50)$ & $2(25)$ & 0.26 \\
\hline
\end{tabular}

Values in parentheses are percentages. ASA $=$ American Society of Anesthesiologist classification; MPI = Mannheim Peritonitis Index.

\section{Hospital Stay}

The median postoperative hospital stay for hospital survivors after $\mathrm{HP}$ and PA was 22 days ( \pm 22 ; range $6-$ 120 ) and 13 days ( \pm 18 ; range $6-112$ ) respectively. The median hospital stay was significantly longer after HP compared to PA $(p<0.001)$. Median stay at the intensive care unit was also significantly longer for HP (2 days \pm 10 ; range $0-61)$ than for PA ( 1 day \pm 8 ; range $0-56)(\mathrm{p}=$ $0.002)$. 


\section{Discussion}

The prevalence of diverticulosis in Westernized countries is rapidly increasing and so are its complications [11]. Nevertheless, a perforated diverticulitis with generalized purulent and fecal peritonitis occurs less frequently. Therefore, recommendations in treating this stage of disease are based on small or retrospective studies. This multicenter report is the largest in the current literature including 200 consecutive patients with acute perforated diverticulitis.

As emergency surgeries for perforated diverticulitis may be associated with substantial morbidity and mortality, optimization of its treatment is important. Primary resection has become the standard practice for patients with generalized peritonitis complicating diverticulitis, but fear of anastomotic leakage often deters many surgeons from performing PA. A diverting stoma or intraoperative colon lavage seems to diminish the number of anastomotic leakages [12]. This management by fear is supported by an experiment of Hawley [13], demonstrating that fecal soiling or peritoneal sepsis may impair the healing of a colonic anastomosis. This is however not supported by clinical experience [12, 14, 15]. Gooszen et al. [5] evaluated the relationship between severity of disease and the patient's general condition to adverse outcomes of PA and observed that the rate of anastomotic leakage was not related to a higher MPI, APACHE II score or the Hinchey score. Outcome of purulent or fecal peritonitis was reported to depend mainly on preoperative fluid resuscitation and antimicrobial therapy, but not on primary repair $[16,17]$. Although not proven in randomized controlled trials, it may seem that improvements in surgical and radiological intervention techniques, anesthesia and intensive care medicine could favor colonic resection with PA in emergency surgery for diverticular disease even if it is complicated by purulent or fecal peritonitis $[5,6,8,18]$.

The mortality rate and rate of complications that needed radiological or operative reinterventions in this study was high (both 27\%), which reflects the severity of the disease and the poor general condition of the patients. In this study significantly higher severity scores (Hinchey score and MPI) were observed in the patients who underwent HP compared to the patients with PA. Besides that, patients of the HP group were of older age and had more comorbidities (higher ASA classification). Therefore, it seems that the more severely affected and high-risk patients underwent an HP. This was also observed in a prospective multicenter study for surgical treatment of sev- eral different complications of diverticular disease [19]. In the latter study, a multifactorial analysis of predictors of HP was made in order to reduce selection bias. Multivariate analysis still showed a twofold increase in postoperative complications after HP compared to PA. There was no significant difference in mortality between the two surgical procedures.

As the operations were classified as emergency and hence may be performed outside office hours, not all procedures were performed by specialist colorectal surgeons. It is known that non-colorectal surgeons prefer HP in case of emergency surgery for colorectal surgery [20]. Besides, an increase in mortality and morbidity is found after emergency surgery performed by a non-colorectal surgeon in such cases [20]. In this study, specialist colorectal surgeons performed significantly more frequently a PA instead of an HP. It also seemed that they had a better outcome than a general surgeon (tables 2,4 ), but when comparing surgeons it is necessary to adjust for differences in case mix and type of surgery, as the best surgeon may get the most difficult job [21]. In contrast with surgeon's experience, the time of operation did not influence the choice of surgical procedure in this study.

In this study, that exclusively covers patients with acute colon perforation due to diverticulitis, a multivariate logistic regression analysis was performed to reduce the found bias in patient selection and surgeon's experience in order to make a comparison between the results after HP and PA. After adjusting for age, ASA, MPI, Hinchey score, and surgeon's experience, mortality was not significantly different between HP and PA. As age, ASA classification and MPI are significant predictors of mortality (table 3) after perforated diverticulitis; it is suggested that further reduction in mortality will require improvement in medical management of pre- and perioperative sepsis and comorbid conditions. Although this is a retrospective study, the analysis has found to be appropriate and therefore the conclusions can be sound.

MPI, ASA classification, age, and Hinchey score could decisively affect postoperative morbidity [22-24]. This is in accordance with the results of this study for ASA classification, Hinchey score and MPI (table 4). It is important that the surgical technique should not be a risk factor for severe postoperative morbidity. This study suggests that PA should not be regarded as an inferior procedure compared to HP, as patients who underwent HP seemed to have more postoperative complications that needed radiological or surgical reintervention, especially when not performed by a specialist colorectal surgeon. These findings are in agreement with the results of other reports $[8$, 
$12,19]$. The difference in number of reinterventions is not necessarily associated to a more advanced disease stage or more comorbidity of the patients in the HP group, as is shown in table 5 . Besides a high complication rate when performing an HP, the reversal of HP is also known to be associated with substantial morbidity (9.1\%) and even mortality (1.7\%) [25]. This is one of the reasons that HP often results in a permanent colostomy [26].

Anastomotic leakage was found in $5 \%$ of the patients. This is in correspondence with the existing literature [15]. Whether or not a diverting stoma prevents against postoperative complications after PA for treatment of perforated diverticulitis remains unclear, as the groups were too small to compare in a multivariate analysis. In rectal cancer a diverting stoma seems to reduce the rate of anastomotic leakage that requires surgical intervention, rather than it protecting against the occurrence of leaks [27]. Finally, total hospital and intensive care unit stay seems to be in favor of PA compared to HP, but again the existing bias in patient selection probably is an important reason for this difference and therefore precludes clinical extrapolation.

In conclusion, this study shows that selected patients with acute perforated diverticulitis can be managed well by PA, as it seems not to be inferior to HP in terms of severe postoperative complications that need surgical or radiological reintervention and mortality. This decision should be made while taking into account the patient's concomitant diseases, response on preoperative resuscitation and the availability of a surgeon experienced in colorectal surgery and intensive care medicine. A prospective randomized trial to compare HP and PA with or without diverting stoma for treatment of perforated diverticulitis with generalized peritonitis is needed to confirm this last statement.

\section{References}

1 Painter NS, Burkitt DP: Diverticular disease of the colon, a 20th century problem. Clin Gastroenterol 1975;4:3-22.

$>2$ Kang JY, Hoare J, Tinto A, Subramanian S, Ellis C, Majeed A, Melville D, Maxwell JD: Diverticular disease of the colon - on the rise: a study of hospital admissions in England between 1989/1990 and 1999/2000. Aliment Pharmacol Ther 2003;17:1189-1195.

-3 Parks TG: Natural history of diverticular disease of the colon. A review of 521 cases. BMJ 1969;4:639-642.

4 Desai DC, Brennan EJ Jr, Reilly JF, Smink RD Jr: The utility of the Hartmann procedure. Am J Surg 1998;175:152-154.

5 Gooszen AW, Tollenaar RA, Geelkerken RH, Smeets HJ, Bemelman WA, Van Schaardenburgh P, Gooszen HG: Prospective study of primary anastomosis following sigmoid resection for suspected acute complicated diverticular disease. Br J Surg 2001;88:693697.

6 Schilling MK, Maurer CA, Kollmar O, Büchler MW: Primary versus secondary anastomosis after sigmoid colon resection for perforated diverticulitis (Hinchey stage III and IV): a prospective outcome and cost analysis. Dis Colon Rectum 2001;44:699703.

-7 Hoemke M, Treckmann J, Schmitz R, Shah S: Complicated diverticulitis of the sigmoid: a prospective study concerning primary resection with secure primary anastomosis. Dig Surg 1999;16:420-424.

$\checkmark 8$ Wedell J, Banzhaf G, Chaoui R, Fischer R, Reichmann J: Surgical management of complicated colonic diverticulitis. Br J Surg 1997; 84:380-383.
9 Billing A, Frohlich D, Schildberg FW: Prediction of outcome using the Mannheim Peritonitis Index in 2003 patients. Br J Surg 1994;81:209-213.

10 Hinchey EJ, Schaal PGH, Richards MB: Treatment of perforated diverticulitis of the colon. Adv Surg 1978;12:85-105.

11 Makela J, Kiviniemi H, Laitinen S: Prevalence of perforated sigmoid diverticulitis is increasing. Dis Colon Rectum 2002;45:955961.

12 Salem L, Flum DR: Primary anastomosis or Hartmann's procedure for patients with diverticular peritonitis? A systematic review. Dis Colon Rectum 2004;47:1953-1964.

13 Hawley PR: Infection: the cause of anastomotic breakdown: an experimental study. Proc R Soc Med 1970;63:752.

14 Tornqvist A, Forsgren A, Faldt K, Jiborn H, Zederfeldt B: Bacterial load and inflammatory reaction in the bowel wall after colonic obstruction. An experimental study in rats. Eur J Surg 1991;157:539-542.

15 Will-Jorgensen P, Guenaga KF, Matos D, Castro AA: Preoperative mechanical bowel cleansing or not? An updated meta-analysis. Colorectal Dis 2005;7:304-310.

16 Gutman M, Klausner JM, Lelcuk S: Fecal peritonitis - the effect on anastomotic healing. Eur Surg Res 1993;25:366-369.

17 Nespoli A, Ravizzini C, Trivella M, Segala M: The choice of surgical procedure for peritonitis due to colonic perforation. Arch Surg 1993;128:814-818.

18 Richter S, Lindemann W, Kollmar O, Pistorius GA, Maurer CA, Schilling MK: Onestage sigmoid colon resection for perforated diverticulitis (Hinchey stages III and IV). World J Surg 2006;30:1027-1032.
9 Constantinides VA, Tekkis PP, Senapati A: Prospective multicentre evaluation of adverse outcomes following treatment for complicated diverticular disease. Br J Surg 2006; 93:1503-1513.

20 Zorcolo L, Covotta L, Carlomagno N, Bartolo DC: Towards lowering morbidity, mortality, and stoma formation in emergency colorectal surgery: the role of specialization. Dis Colon Rectum 2003;46:1461-1468.

21 Copeland GP, Sagar P, Brennan J, Roberts G, Ward J, Cornford P, Millar A, Harris C: Riskadjusted analysis of surgeon performance: a one-year study. Br J Surg 1995;82:408-411.

22 Makela J, Kiviniemi H, Laitinen S: Prognostic factors of perforated sigmoid diverticulitis in the elderly. Dig Surg 2005;22:100-106.

23 Pisanu A, Cois A, Uccheddu A: Surgical treatment of perforated diverticular disease: evaluation of factors predicting prognosis in the elderly. Int Surg 2004;89:35-38.

24 Biondo S, Ramos E, Deiros M, Rague JM, De Oca J, Moreno P, Farran L, Jaurrieta E: Prognostic factors for mortality in left colonic peritonitis: a new scoring system. J Am Coll Surg 2000;191:635-642.

-25 Aydin HN, Remzi FH, Tekkis PP, Fazio VW Hartmann's reversal is associated with high postoperative adverse events. Dis Colon Rectum 2005;48:2117-2126.

$\checkmark 26$ Banerjee S, Leather AJM, Rennie JA, Samano N, Gonzalez JG, Papagrigoriadis S: Feasibility and morbidity of reversal Hartmann's. Colorectal Dis 2005; 7:454-459.

27 Gastinger I, Marusch F, Steinert R, Wolff S, Koeckerling F, Lippert H: Protective defunctioning stoma in low anterior resection for rectal carcinoma. Br J Surg 2005;92:15651566. 